\section{Safety of using direct oral anticoagulants in the diagnostic workup of outpatients with suspicion of acute venous thromboembolism}

In patients with a clinical suspicion of venous thromboembolism (VTE), guidelines suggest treatment with parenteral anticoagulants during the diagnostic workup, as opposed to no treatment, if diagnostic tests are expected to be delayed. ${ }^{1}$ Many patients can be safely managed as outpatients, if they meet particular criteria. ${ }^{2}$ Although low molecular weight heparin (LMWH) has been a standard of care for the management of these patients, direct oral anticoagulants (DOAC) are becoming increasingly used in the treatment of patients with suspected VTE. To date, no study has analysed the safety of DOAC during the diagnostic workup period.

The Ottawa Hospital Thrombosis Program provides assessment of outpatients suspected of VTE 363 days of the year. Patients presenting to any emergency room in the Ottawa area with symptoms suggestive of deep vein thrombosis (DVT) and pulmonary embolism (PE), or to a walk-in clinic or primary care practice with symptoms of DVT, can be urgently referred and seen within 24 hours for diagnostic management, provided that they are initiated on full-dose anticoagulation with $\mathrm{LMWH}$ or DOAC while awaiting testing. Our criteria for outpatient management includes absence of cardiopulmonary compromise, absence of contraindications to $\mathrm{LMWH}$ or DOAC, creatinine clearance $>30 \mathrm{~mL} / \mathrm{min}$, platelets $>50$ $\mathrm{g} / \mathrm{L}$, no unexplained severe anemia, no recent or active bleeding, and logistical feasibility (accessibility to hospital, no need for parenteral medication). Treatment with a DOAC is contraindicated in both pregnancy and patients weighing above $120-140 \mathrm{~kg}$.

In this study, our objective was to assess the rate of major bleeding in outpatients treated with DOAC during the diagnostic workup of VTE.

This was a retrospective chart review evaluation of outpatients with suspicion of VTE, referred to The Ottawa Hospital Thrombosis Program between January 1, and March 31, 2018. Patients were referred from either the emergency department, or a general practitioner. Suspected VTE included either lower or upper extremity deep vein thrombosis, or pulmonary embolism. Data was collected by reviewing the medical records of patients referred to the thrombosis clinic. Adult patients seen in the thrombosis clinic and treated with anticoagulation while awaiting results of diagnostic workup were included. The medical records of patients who did not attend their appointment at the thrombosis clinic were analyzed to ensure no adverse events. Patients, who already had a confirmed diagnosis of VTE before attending the clinic, or were not given anticoagulants during the workup period, were not included.

The following data was collected for each patient: 1) Patient demographics (age, sex, body weight), 2) VTE risk factors (recent surgery, cancer, immobilization, pregnancy), 3) date/time of suspicion of VTE and date/time of

Table 1. Characteristics of the patients according to exposure to low molecular weight heparin or direct oral anticoagulants.

\begin{tabular}{|c|c|c|c|c|}
\hline Characteristics & $\begin{array}{c}\text { All patients } \\
(n=173)\end{array}$ & $\begin{array}{c}\text { DOAC patients } \\
(n=95)\end{array}$ & $\begin{array}{l}\text { LMWH patients } \\
\quad(n=78)\end{array}$ & $P$ \\
\hline Age (years) Median (range) & $56(18-95)$ & $59(19-95)$ & $51(18-91)$ & 0.01 \\
\hline Weight $(\mathrm{kg})$ Median (range) ${ }^{*}$ & $81(43.6-183)$ & $81.6(45.3-132)$ & $79.7(43.6-183)$ & 0.67 \\
\hline Male Sex, n (\%) & $65(37.6)$ & $38(40.0)$ & $27(37.6)$ & 0.47 \\
\hline Rule out PE, n (\%) & $79(45.7 \%)$ & $42(44.2)$ & $37(47.4)$ & 0.67 \\
\hline \multicolumn{5}{|l|}{ Risk factors for VTE } \\
\hline Cancer, n (\%) & $36(20.8)$ & $11(11.6)$ & $25(32.1)$ & 0.001 \\
\hline Immobilization, n (\%) & $18(10.4)$ & $11(11.6)$ & $7(9.0)$ & 0.58 \\
\hline Recent surgery, n (\%) & $14(8.1)$ & $10(10.5)$ & $4(5.1)$ & 0.20 \\
\hline Pregnancy/post-partum & $9(5.2)$ & $1(1.0)$ & $8(10.3)$ & 0.02 \\
\hline \multicolumn{5}{|l|}{ Comorbidities } \\
\hline Chronic cardiac insufficiency, n (\%) & $4(2.3)$ & $2(2.1)$ & $2(2.6)$ & 1 \\
\hline Respiratory insufficiency, n (\%) & $11(6.4)$ & $4(4.2)$ & $7(9.0)$ & 0.23 \\
\hline Liver insufficiency, n (\%) & $2(1.2)$ & $1(1.1)$ & $1(1.3)$ & 01 \\
\hline Creatinine $(\mu \mathrm{mol} / \mathrm{L})$, Median (range) ${ }^{* *}$ & $71.5(29-256)$ & $75.5(44-135)$ & $65(29-256)$ & 0.01 \\
\hline Creatinine clearance $(\mathrm{mL} / \mathrm{min})$ Median (range) ${ }^{* * *}$ & $103.4(24.6-284.3)$ & $89.8(30.6-238.7)$ & $124.9(24.6-284.3)$ & 0.01 \\
\hline Hemoglobin Median (range) & $131(34-173)$ & $137(62-173)$ & 127 (34-157) & 0.001 \\
\hline Platelets Median (range) & $224(75-547)$ & $234(75-547)$ & $218(90-509)$ & 0.56 \\
\hline "Likely" pre-test probability, n (\%) & $56(32.4)$ & $28(29.5)$ & $28(35.9)$ & 0.37 \\
\hline History of major bleeding, n (\%) & $3(1.7)$ & $2(2.1)$ & $1(1.3)$ & 1 \\
\hline VTE confirmed, n (\%) & 86 (49.7) & $40(42.1)$ & $46(59.0)$ & 0.03 \\
\hline Major bleeding \% (95\% CI) & $0(0-2.2)$ & $0(0-3.9)$ & $0(0-4.8)$ & \\
\hline CRNMB, $\mathrm{n}$ & $2(1.2)$ & $2(2.1)$ & 0 & 0.50 \\
\hline CRNMB, \% $(95 \% \mathrm{CI})$ & $1.2 \%(0.3-4.1)$ & $2.1 \%(0.6-7.4)$ & $0 \%(0-4.7)$ & \\
\hline
\end{tabular}

DOAC: direct oral anticoagulants; LMWH: low molecular weight heparin; VTE: venous thromboembolism; CRNMB: clinically relevant non-major bleed; * 159 patients; ** 166 patients; ***154 patients; CI: confidence interval. 


\author{
540 add-on visits \\ 367 patients excluded \\ - New thrombosis confirmed \\ prior to visit $(n=116)$ \\ Did not receive anticoagulants \\ prior to visit $(n=17)$ \\ - Other $(n=234)$
}

173 patients with suspicion of venous thromboembolism imaging tests, 4) bleeding events, 5) bloodwork (hemoglobin, platelets, creatinine), 6) anticoagulant used (LMWH vs. DOAC) and dose, 7) presence of co-morbidities (liver, heart or lung insufficiency). The Wells Criteria for PE or DVT were assessed and used by the emergency physicians or primary care providers to determine the pre-test probability of VTE.

The primary study outcome was to identify and compare the rate of major bleeding events, as defined by the International Society on Thrombosis and Hemostasis (overt bleeding associated with a decrease in hemoglobin of $2 \mathrm{~g} / \mathrm{dL}$ or more or that required a transfusion of two or more units of blood, occurred in a critical site, or contributed to death) between DOAC and LMWH during VTE workup. ${ }^{3}$ The International Society of Thrombosis and Haemostasis criteria for clinically relevant non-major bleed (CRNMB) were also used (i.e. overt bleeding that did not meet the criteria for major bleeding but was associated with the need for medical intervention, contact with a physician, or interruption of the study drug or with discomfort or impairment of activities of daily life. ${ }^{4}$

Research ethics board approval was obtained to review hospital charts of consecutive, eligible patients. Baseline summary statistics were reported as median and range for continuous variables and as number and proportions for categorical variables. Comparisons were made by Chi-square test or Fisher exact test for qualitative variables and Wilcoxon rank-sum test for quantitative variables, which were not normally distributed. Statistical analyses were performed using Stata 14.2 (StataCorp; 4905 Lakeway Dr. College Station, TX, USA).

Of the 540 charts reviewed, 367 patients were excluded for various reasons: 116 had a diagnosis of VTE confirmed before the thrombosis clinic appointment, 17 were not given anticoagulant medication during the diagnostic workup, and 234 were referred for other reason than acute DVT or PE (Figure 1). A total of 173 patients with suspected VTE were included in the analyses. The median age of participants was 56 years (range: 18-95), $65(37.6 \%)$ were men (Table 1). Ninety-five patients $(54.9 \%)$ were treated with a DOAC, and $78(45.1 \%)$ with LMWH during the diagnostic workup period. Two patients $(1.1 \%)$ did not attend their scheduled appointment. Patients treated with a DOAC had a "likely" pretest probability of VTE $29.5 \%$ of the time $(n=28)$ with confirmed VTE in $42.1 \%(n=40)$. Patients treated with
LMWH had a "likely" pre-test probability of VTE $35.9 \%$ of time $(n=28)$ with VTE confirmed in $59 \%(n=46)$. The mean value for hemoglobin was significantly lower in the patients prescribed LMWH $(P=0.001)$. LMWH was the preferred treatment in cancer patients $(P=0.001)$, and as expected in the pregnancy/postpartum period.

No patients ( $0 \% ; 95 \%$ confidence interval [CI]): $0-2.2)$ experienced major bleeding from treatment initiation to diagnostic test, including the two patients who did not attend their next day appointment. Estimates of incidence rates of major bleeding in patients treated with DOAC or LMWH were $0 \%$; $95 \%$ CI: $0-3.9$ and $0 \%$; $95 \%$ CI: $0-4.8$ respectively $(P=$ not significant $[\mathrm{NS}])$. Two $(2.1 \%$; 95\% CI: $0.6-7.4)$ patients treated with a DOAC experienced CRNMB compared with $0 \%(95 \%$ CI: $0-4.7)$ $(n=0)$ in patients treated with $\mathrm{LMWH}(P=0.50)$. No treatment failure (i.e. no patient experienced new or worsening VTE symptoms) was observed (0\%; 95\% CI: 0-2.2).

In this population, outpatient management of patients with a suspicion of VTE appeared safe regardless of the choice of anticoagulant therapy. None of the patients treated with DOAC or LMWH experienced major bleeding. CRNMB was very infrequent, and although experienced by two patients receiving a DOAC and none receiving $\mathrm{LMWH}$, the difference was not significant. Due to the retrospective nature of the study, we cannot exclude information bias, such as an excess of reporting adverse events in patients receiving a DOAC compared with LMWH.

More cancer patients received LMWH than DOAC during the diagnostic workup of VTE. Clinical guidelines on the management of VTE in cancer patients have been widely implemented and physicians probably tended to apply these guidelines. This probably also explains why LMWH patients had a lower median level of hemoglobin. Pregnancy is a contraindication for management with a DOAC, and this was reflected in the results. Patients treated with LMWH generally had higher creatinine clearance values. This is likely because the creatinine clearance calculation is weight-based. Patients treated with LMWH tended to be heavier, as use of DOAC for patients' weighing above $120-140 \mathrm{~kg}$ is not recommended. ${ }^{5}$

Our results apply to a specific patient population eligible for outpatient management (i.e. at low bleeding risk). They were also assessed clinically prior to referral and 
were tested for d-dimer when appropriate. This may explain the relatively high proportion of patients with a likely pre-test probability and a high proportion of confirmed VTE.

Our study has several limitations deserving comments. i) This was a retrospective study. However, we checked the outcome of patients who did not attend their clinic appointment $(n=2)$ to ensure no adverse events. Furthermore, to the best of our knowledge, this is the first study reporting on this issue and our result bring valuable information on anticoagulation with DOAC in outpatient management of suspected VTE. ii) The sample size was relatively small. We analyzed three consecutive months as this is our limit for record keeping for patients who do not attend the clinic. Data on these patients is crucial to validate the safety of DOAC use in outpatients. The upper limit of the CI for the observed rate of major bleeding events appears clinically reasonable and supports using DOAC in this indication. Evidence for using LMWH in this setting is also scarce and based on few patients. ${ }^{2,6}$

In conclusion, our study showed the safety of using DOAC during the diagnostic workup of VTE in outpatients. Future prospective studies are needed to confirm our findings.

\section{Maddie S. Stephen, Lana Castellucci, Marc Carrier,} Lisa Duffett, Gregoire Le Gal, Marc Rodger, Philip S. Wells and Aurélien Delluc

Department of Medicine, Ottawa Hospital Research Institute at the University of Ottawa, Ottawa, Ontario, Canada.

Correspondence: AURELIEN DELLUC adelluc@toh.ca

doi:10.3324/haematol.2019.232884

Funding: Dr. Le Gal holds an Early Researcher Award from the
Province of Ontario; a Heart and Stroke Foundation of Canada (HSFC) Ontario Mid-Career Investigator Award; and the Chair on Diagnosis of Venous Thromboembolism from the Department of Medicine and the University of Ottawa. Dr. Rodger holds a Research Chair from the Department of Medicine and the University of Ottawa. The views expressed in this article are those of the author(s) and not necessarily those of the NHS, the NIHR, or the Department of Health.

Information on authorship, contributions, and financial \& other disclosures was provided by the authors and is available with the online version of this article at www. haematologica.org.

\section{References}

1. Kearon C, Akl EA, Comerota AJ, et al. Antithrombotic therapy for VTE disease: antithrombotic therapy and Prevention of thrombosis, $9^{\text {th }}$ ed: American College of Chest Physicians evidence-based clinical practice guidelines. Chest. 2012;141(2 Suppl):e419S-494S.

2. Siragusa S, Anastasio R, Porta C, et al. Deferment of objective assessment of deep vein thrombosis and pulmonary embolism without increased risk of thrombosis: a practical approach based on the pretest clinical model, D-dimer testing, and the use of low-molecular-weight heparins. Arch Intern Med. 2004;164(22):2477-2482.

3. Schulman S, Kearon C. Definition of major bleeding in clinical investigations of antihemostatic medicinal products in non-surgical patients. J Thromb Haemost. 2005;3(4):692-694.

4. Kaatz S, Ahmad D, Spyropoulos AC, Schulman S. Definition of clinically relevant non-major bleeding in studies of anticoagulants in atrial fibrillation and venous thromboembolic disease in non-surgical patients: communication from the SSC of the ISTH. J Thromb Haemost. 2015;13(11):2119-2126.

5. Martin K, Beyer-Westendorf J, Davidson BL, Huisman MV, Sandset PM, Moll S. Use of the direct oral anticoagulants in obese patients: guidance from the SSC of the ISTH. J Thromb Haemost. 2016;14(6):1308-1313.

6. Imberti D, Ageno W, Dentali F, Giorgi Pierfranceschi M, Croci E, Garcia D. Management of primary care patients with suspected deep vein thrombosis: use of a therapeutic dose of low-molecular-weight heparin to avoid urgent ultrasonographic evaluation. J Thromb Haemost. 2006;4(5):1037-1041. 